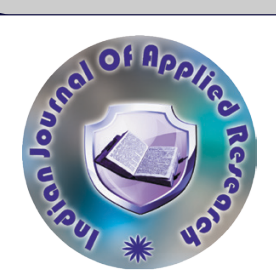

\title{
Oncology
}

\section{RATIO OF SERUM CA125 TO RADIOLOGICAL PERITONEAL CARCINOMATOUS INDEX AS A NOVEL MARKER OF RESPONSE TO NEO ADJUVANT CHEMOTHERAPY AND PREDICTIVE MODEL OF SURGICAL OUTCOME IN ADVANCED EPITHELIAL OVARIAN CANCER-A SINGLE INSTITUITIONAL STUDY.}

\begin{tabular}{l|}
\hline Dr Prem Kumar \\
Devdoss \\
\hline Dr Prasanna \\
Srinivasa Rao H* \\
\hline Dr N. \\
Roobalakshmi \\
\hline
\end{tabular}

MD DM Associate Professor of Medical Oncology Govt. Arignar Anna Memorial Cancer Hospital \& Research Institute, Kanchipuram.

MS MCh Assistant Professor of Surgical Oncology, Govt. Arignar Anna Memorial Cancer Hospital \& Research Institute, Kanchipuram. *Corresponding Author

DM RD Senior Resident in Radiology Govt. Arignar Anna Memorial Cancer Hospital \& Research Institute, Kanchipuram.

\section{ABSTRACT}

Objective: The objective of this retrospective study is to develop a novel marker- ratio of serum CA 125 to peritoneal carcinomatous index(PCI) - to predict the response in women receiving neoadjuvant chemotherapy for newly diagnosed advanced epithelial ovarian cancer at our centre.

Methods: Medical records of women who were newly diagnosed with inoperable advanced ovarian cancer stages III and IV at our centre were selected. Only people with completely documented records in the years 2017 \& 2018 were selected. Only patietns with serous histology were chosen. Pre chemotherapy serum CA125 value was noted. Radiological PCI was calculated by reviewing the CECT films \& reports of the patients.

Patients were compared with the ratio of CA125 to radiological PCI and clinical \& pathological response to neo adjuvant chemotherapy. All patients received standard doses of three weekly Paclitaxel and Carboplatin based chemotherapy. Based on the ratio of CA125 to PCI patients were divided into 2 groups - ratio more than 100 and less than 100

Results: A total of 34 were patients were found to meet the eligible criteria. Response assessment was done after 3 to 4 cycles of neoadjuvant chemotherapy. The overall response rate to neoadjuvant chemotherapy in patients in group 1 (CA125/PCI ratio > 100) was significantly higher as compared to patients in group $2(\mathrm{CA} 125 / \mathrm{PCI}$ ratio $<100)$.

Conclusion: In summary, CA 125 to PCI ratio is novel method to predict response to chemotherapy in advanced epithelial ovarian cancers. This value is a helpful measurement that allows the clinicians to measure the degree of chemosensitivity prior to cytoreductive surgery. This measures the inherent tumor biology and to aids in surgical decision making regarding the role and extent of cytoreduction as well as alternate systemic/local therapies.

\section{KEYWORDS : Advanced ovarian cancer, CA125, Peritoneal Carcinomatous Index, PCI, ratio}

\section{Background:}

Ovarian cancer is the third most common cancer in women and the second most common cause of cancer-related deaths from gynaecologic malignancies worldwide (1). Incidences are higher in developed countries and among postmenopausal women (2). Symptoms for ovarian cancer are nonspecifific, causing to it being diagnosed when it has already developed into an advanced International Federation of Gynaecology and Obstetrics (FIGO) stage and tumor cells have already disseminated into the peritoneal cavity and to other organs(3)(4).

Intraperitoneal spread of ovarian cancer is the most typical, earliest distribution pathway (5).Epithelial ovarian cancer (EOC) is the most lethal gynaecologic malignancy and is closely associated with tumor recurrence and chemoresistance (6). Approximately $70-75 \%$ of patients are diagnosed at an advanced stage with widespread peritoneal lesions and frequent chemoresistance (7).

At present, the gold standard for management is primary debulking surgery followed by platinum-based adjuvant chemotherapy. However, optimal debulking can be achieved in only $30-60 \%$ of stage III/IV ovarian cancers $(8)(9)(10)(11)$

Neoadjuvant chemotherapy(NACT) followed by interval debulking surgery has been proposed as an alternative to conventional Primary cytoreduction. These indications are for patients with a low probability of optimal cytoreduction or reversible contraindications

A variety of biomarkers have been developed to monitor growth of ovarian cancer and to detect disease at an early interval. Amongst them CA125 has been the most extensively studied and clinically utilized serum tumor marker. As a clinical tool, prognostic markers like CA125 may potentially help individualizing treatment within subgroups of patients. Serum levels of CA125 are used to monitor responses to chemotherapy, relapse, and disease progression in ovarian cancer patients.(12)

Levels of CA125 can be elevated in the serum before clinical development of primary and recurrent ovarian carcinoma . (13) The CA 125 elimination half life can be used as a measure of chemotherapy sensitivity in women with newly diagnosed advanced epithelial ovarian cancer who are being treated with neoadjuvant chemotherapy.

Identifying predictive markers of chemosensitivity at diagnosis or during the initial treatment phase may allow oncologists to tailor systemic and surgical treatments in the first line setting. Various groups have examined the significance of Ca125 in women with advanced high grade serous ovarian carcinoma being treated with primary cytoreductive surgery .(14)

Ca125 expression is believed to correlate with tumor burden, therefore a decline in Ca125 often indicates reduction in residual disease. Even for the majority of patients who attained optimal debulking after NACT, due to differences in chemosensitivity, patient outcomes remain varied.

However, a significant proportion of patients cannot be optimally cytoreduced even after NACT-Interval Debulking Surgery(IDS) and this leads to the morbidity of surgery with no expected survival benefit (15)(16)(17). Single variables have been combined into predictive cytoreduction models to improve accuracy in the settings of PDS (18) and recurrent disease (19)(20).

Peritoneal carcinomatosis index(PCI) was initially developed for colonic cancers and other gastro intestinal cancers for estimating the volume of peritoneal dissemination. The gold standard method of calculating PCI is by surgical methods. The radiological calculation by CT or MRI scans have the limitations of inter-observer variation and erring on either side particularly for lesions close to the mesentery and small lesions. Nevertheless measuring PCI by radiological means is a cost effective, resource less intense and non invasive procedure.

The peritoneal cancer index (PCI) is used to assess the extent of peritoneal cancer throughout the peritoneal cavity. For this purpose, the peritoneal cavity is divided in 13 well-defined regions (see figure). 
In each of the 13 regions, the size of the largest tumor nodule is measured. If no tumor is visualized, a score of " 0 " is given to that region. If the largest tumor nodule is smaller than $0.5 \mathrm{~cm}$, the score is " 1 ". For tumors measuring between $0.5 \mathrm{~cm}$ and $5 \mathrm{~cm}$, the score is " 2 ". For lesions larger than $5 \mathrm{~cm}$, the score is " 3 ". If there is layering or a confluence of multiple small tumor nodules, the score is " 3 ". The PCI is calculated by adding the scores of all 13 regions together with a maximum score of 39 .

\section{Figure 1 : Calculation of PCI}
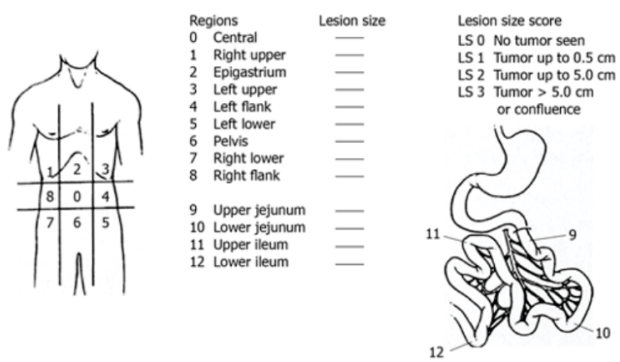

In our study we try to study the chemo sensitiveness and hence the tumour biology of advanced ovarian cancer by bringing both the biochemical and volume parameters as a single novel ratio.

\section{Methods:}

Medical records of women who were newly diagnosed with inoperable advanced ovarian cancer stages III and IV at our centre were selected. Only people with completely documented records in the years 2017 \& 2018 were selected. Clinical information on age, menopausal status, BMI, radiological PCI, stage, histology, chemotherapy drugs used, CA 125 levels before and after neoadjuvant chemotherapy were noted and information analysed.

Patients more than 70 years, incomplete data, receiving non standard chemotherapy \& non serous histology were not included in the study.

Radiological assessment and CA 125 levels were repeated after 3 or 4 cycles of neoadjuvant chemotherapy three weekly paclitaxel plus carboplatin. Patients with good radiological response and amenable to cytoreductive surgery were taken up for surgery. The findings at surgery and the pathological response to chemotherapy were noted.

Based on the ratio of CA125 to PCI patients were divided into 2 groups -ratio more than 100 and less than 100 .

\section{Results:}

The data of thirty four patients were analysed. The table shows the comparison between the two groups based on the CA 125/ PCI ratio. Group 1 ratio $>100$, Group 2 ratio $<100$.

Optimal cytoreduction was achieved in 19/21 patients in group 1 as against 7/13 patients in group 2 ( $p$ value 0.014 ). Clinical \& pathological complete response was comparatively higher in group 1 but statistical significance could not be proven ( $\mathrm{p}$ value $0.079 \& 0.091$ respectively)

Table 1: CA 125 / radiological PCI ratio and patient parameters:

\begin{tabular}{|l|c|c|c|}
\hline CA125/PCI ratio & $\begin{array}{c}\text { Group 1 } \\
>\mathbf{1 0 0}\end{array}$ & $\begin{array}{c}\text { Group 2 } \\
<\mathbf{1 0 0}\end{array}$ & $\begin{array}{c}\text { P } \\
\text { value }\end{array}$ \\
\hline No. of Patients & 21 & 13 & - \\
\hline Age (years) & $62.5(54-69)$ & $63(56-67)$ & - \\
\hline CA 125 (pre chemo) range & $452-13450$ & $398-9048$ & - \\
\hline Radiological PCI & $7-27$ & $8-32$ & - \\
\hline $\begin{array}{l}\text { Interval cytoreductions feasible } \\
\text { after 3-4 cycles }\end{array}$ & 19 & 7 & $\mathbf{p}=\mathbf{0 . 0 1 4}$ \\
\hline $\begin{array}{l}\text { Interval cytoreductions feasible } \\
\text { after 6 cycles }\end{array}$ & 2 & 2 & - \\
\hline $\begin{array}{l}\text { Clinical complete response in } \\
\text { cytoreducible cpatients }\end{array}$ & 12 & 2 & $\mathrm{P}=0.079$ \\
\hline Pathological complete response & 9 & 1 & $\mathrm{P}=0.091$ \\
\hline Inoperable/ progressive disease & 0 & 4 & - \\
\hline
\end{tabular}

\section{Discussion:}

Gynecologic oncologists often rely on surrogate indicators including tumor markers and imaging assessment. These assessment tools have inherent limitations and lack precision and accuracy.
Diagnostic laparoscopy provides a more accurate measure of disease volume and disease distribution. Patients with favourable scores on laparoscopic assessment, which are predictive of tumor resectability, undergo cytoreductive surgery. Those with unfavourable scores are not taken for attempted cytoreduction thus avoiding futile laparotomy.

In patients with substantial platinum sensitivity, platinum-based therapy may have a larger impact on survival than the degree of surgical resection at cytoreductive surgery. This finding suggests that tumors that are less sensitive to chemotherapy appear more reliant on the degree of surgical resection where complete resection harbors significant survival advantage. These are the tumors that benefit the most from peritonectomy and HIPEC therapy.

The two most important factors that determine survival \& prognosis in advanced epithelial ovarian cancer is chemosensitivity (platinum sensitivity) and completeness of cytoreduction.

Chemosensitivity is an important predictor of survival in ovarian malignancies. To date, a commonly used indicator of platinum sensitivity is the platinum free interval which measure the time lapse between the last platinum treatment and the date of recurrence. Chemosensitivity depends on the tumour biology.

PCI depicts the gross volume of disease in the entire abdominal cavity. In serous ovarian cancer the CA 125 is secreted in higher quantity facilitating the monitoring of tumour response to chemotherapy biochemically.

This CA125 to PCI ratio brings both these factors into one single variable and easier to analyse the tumour biology accounting both the biology and the volume of the disease.

From our study it is clear that a large volume tumor with low CA125 has inherent chemo resistance. Such patients are reliant on surgical completeness; prolonged surgery, mutlivisceral resection and increased surgical morbidity might be acceptable in favour of improved survival.

However, the role of aggressive surgery and the added benefit harboured by complete cytoreduction in women with favourable score deserves further studying.

This being a retrospective study has its own limitations. The experience and familiarity of the radiologist with measuring radiological PCI is of utmost importance. Significant inter observer variations occur in interpreting PCI by radiological means. Radiological PCI values may significantly differ from PCI values obtained by laparotomy or laparoscopy to a certain extent.

Given all these limitations, still the non invasiveness and cost effectiveness of the procedure scores over.

\section{Conclusion:}

In summary, CA 125 to PCI ratio is novel method to predict response to chemotherapy in advanced epithelial ovarian cancers. This value is a helpful measurement that allows the clinicians to measure the degree of chemo sensitivity prior to cytoreductive surgery. This measures the inherent tumor biology and to aids in surgical decision making regarding the role and extent of cytoreduction as well as alternate systemic/local therapies.

More prospective trials with an arm measuring laparoscopic PCI should be taken up to further qualify our data so as to design a novel and cost effective predictive modality.

\section{References:}

1. Jemal A, Bray F, Center MM, Ferlay J, Ward E, Forman D. Global cancer statistics. CA Cancer J Clin. 2011;61(2):69-90. - Google Search [Internet]. [cited 2020 Jul 27]. Available from:

2. Holschneider CH, Berek JS. Ovarian cancer: Epidemiology, biology, and prognostic factors. Vol. 19, Seminars in Surgical Oncology. 2000. p. 3-10,

3. Heintz AP, Odicino F, Maisonneuve P, et al. Carcinoma of the ovary. FIGO 26th Annual Report on the Results of Treatment in Gynecological Cancer. Int J Gynaecol Obstet. 2006;95(Suppl 1):S161-92.

4. Engel J, Eckel R, Schubert-Fritschle G, Kerr J, Kuhn W, Diebold J, et al. Moderate progress for ovarian cancer in the last 20 years: Prolongation of survival, but no improvement in the cure rate. Eur J Cancer. 2002 Dec;38(18):2435-45.

5. Meyers MA. Distribution of intra abdominal malignant seeding: dependency on dynamics of flow of ascitic fluid. AMERJROENTGENOL [Internet]. 1973 Sep [cited 2020 Jul 27];119(1):198-206. Available from: http:// www. ajronline. org/doi/10.2214/ajr.119.1.198 
6. Lowe KA, Chia VM, Taylor A, O'Malley C, Kelsh M, Mohamed M, et al. An international assessment of ovarian cancer incidence and mortality. Gynecol Oncol. $2013 \mathrm{Jul} ; 130(1): 107-14$

7. Mercieca-Bebber R, Friedlander M, Kok PS, Calvert M, Kyte D, Stockler M, et al. The patient-reported outcome content of international ovarian cancer randomised controlled trial protocols. Qual Life Res. 2016;25(10):2457-65.-

8. Griffiths CT. Surgical resection of tumor bulk in the primary treatment of ovarian carcinoma. Natl Cancer Inst Monogr. 1975;42:101-4. - Google Search [Internet]. [cited 2020 Jul 27].

9. Chang S-J, Bristow RE, Chi DS, Cliby WA. Role of aggressive surgical cytoreduction in advanced ovarian cancer. J Gynecol Oncol [Internet]. 2015 Oct 1 [cited 2020 Jul 27];26(4):336. Available from: https:// ejgo. org/ DOIx. php?id= 10.3802/ jgo. 2015.26.4.336

10. Dauplat J, Le Bouedec G, Pomel C, Scherer C. Cytoreductive surgery for advanced stages of ovarian cancer. Semin Surg Oncol. 2000;19(1):42-8. Gao et al. Journal of Ovarian Research (2019) 12:85 Page 7 of 88. Lee YJ, Chung YS, Lee JY, Nam EJ, Kim SW, Kim S, et al. Impact of increased utilization of neoadjuvant chemotherapy on survival in patients with -

11. Tseng JH, Cowan RA, Zhou Q, Iasonos A, Byrne M, Polcino T, et al. Continuous improvement in primary Debulking surgery for advanced ovarian cancer: do increased complete gross resection rates independently lead to increased progression-free and overall survival? Gynecol Oncol. 2018;151(1):24-31.

12. O'Brien TJ, Beard JB, Underwood LJ, Dennis RA, Santin AD, York L: The CA 125 gene: an extracellular superstructure domi nated by repeat sequences. Tumour Biol 2001, 22:348-366.

13. Burger RA, Darcy KM, DiSaia PJ, Monk BJ, Grosen EA, Gatanaga T, Granger GA, Wang J, Tian C, Hanjani P, Cohn DE: Association between serum levels of soluble tumor necrosis factor receptors/CA 125 and disease progression in patients with epithelial necrosis factor receptors/CA 125 and disease progression in patients with epithelial

14. May T, Stewart JM, Bernardini MQ, Ferguson SE, Laframboise S, Jiang H, et al. The
Marian malignancy: a gynecologic oncology group study. Cancer 2004, 101:106-115.May T, Stewart JM, Bernardini MQ, Ferguson SE, Laframboise S, Jiang H, et al. The
prognostic value of perioperative, pre-systemic therapy CA125 levels in patients with high-grade serous ovarian cancer. Int J Gynecol Obstet. $2018 \mathrm{Feb}$ 1;140(2):247-52.

15. Tangjitgamol S, Manusirivithaya S, Laopaiboon M, Lumbiganon P, Bryant A. Interval debulking surgery for advanced epithelial ovarian cancer. Cochrane Database Syst Rev. 2016; no. 1, p. CD006014, Valadares CV, Baiocchi G, Mantoan H, Saito A, Sanches S, et al. Neoadjuvant chemotherapy followed by interval Debulking surgery and the risk of platinum resistance in epithelial ovarian Cancer. Ann Surg Oncol. 2015;22(Suppl 3):S971-8. -

16. Pepin K, Bregar A, Davis M, Melamed A, Hinchcliff E, Gockley A, et al. Intensive care admissions among ovarian cancer patients treated with primary debulking surgery and neoadjuvant chemotherapy-interval debulking surgery. Gynecol Oncol. 2017 Dec $1 ; 147(3): 612-6$

17. Aletti GD, Dowdy SC, Podratz KC, Cliby WA. Relationship among surgical complexity, short-term morbidity, and overall survival in primary surgery for advanced ovarian cancer. Am J Obstet Gynecol [Internet]. 2007 [cited 2020 Jul 27];197(6):676.e1-676.e7. Available from: https://pubmed.ncbi.nlm.nih.gov/18060979/

18. Rutten MJ, Van De Vrie R, Bruining A, Spijkerboer AM, Mol BW, Kenter GG, et al Predicting surgical outcome in patients with international federation of gynecology and obstetrics stage III or IV ovarian cancer using computed tomography: A systematic obstetrics stage III or IV ovarian cancer using computed tomography: A systematic
review of prediction models [Internet]. Vol. 25, International Journal of Gynecological review of prediction models [Internet]. Vol. 25, International Journal of Gynecological
Cancer. Lippincott Williams and Wilkins; 2015 [cited 2020 Jul 27]. p. 407-15. Available from: https://pubmed.ncbi.nlm.nih.gov/25695545/

19. Harter P, Sehouli J, Reuss A, Hasenburg A, Scambia G, Cibula D, et al. Prospective validation study of a predictive score for operability of recurrent ovarian cancer: The multicenter intergroup study DESKTOP II. A project of the AGO kommission OVAR, AGO study group, NOGGO, AGO-Austria, and MITO. Int J Gynecol Cancer [Internet]. 2011 [cited 2020 Jul 27];21(2):289-95. Available from: https:// pubmed. ncbi. nlm. nih. gov/21270612/

20. Van de Laar R, Zusterzeel PLM, Van Gorp T, Buist MR, Van Driel WJ, Gaarenstroom $\mathrm{KN}$, et al. Cytoreductive surgery followed by chemotherapy versus chemotherapy alone for recurrent platinum-sensitive epithelial ovarian cancer (SOCceR trial): A multicenter randomised controlled study. BMC Cancer [Internet]. 2014 Jan 14 [cited 2020 Jul 27];14(1):22. Available from: http:// bmccancer. biomedcentral. com/articles/ 10.1186/ $1471-2407-14-22$ 